Check for updates

Cite this: Phys. Chem. Chem. Phys., 2021, 23, 13087

Received 3rd February 2021, Accepted 24th May 2021

DOI: $10.1039 / \mathrm{d} 1 \mathrm{cp00522g}$

rsc.li/pccp

\title{
Room-temperature diffusion of metal clusters on graphene $\dagger$
}

\author{
Mohammad Zarshenas, (D) *a Victor Gervilla, ${ }^{a}$ Davide G. Sangiovanni (D) ${ }^{b}$ and \\ Kostas Sarakinos (D) ${ }^{a}$
}

\begin{abstract}
We study the diffusion dynamics, the diffusion mechanisms, and the adsorption energetics of Ag, Au, $\mathrm{Cu}$, and Pd dimers, as well as of Ag trimers on single-layer graphene (SLG) by means of ab initio molecular dynamics (AIMD) simulations and density-functional theory (DFT) calculations. The simulations show that $\mathrm{Ag}, \mathrm{Cu}$, and $\mathrm{Au}$ clusters exhibit a super-diffusive pattern characterized by long jumps, which can be explained by the flat potential energy landscape (PEL) (corrugation of a few tens of meV) encountered by those clusters on SLG. Pd dimers, instead, diffuse in a pattern that is reminiscent of conventional random walk, which is consistent with a significantly rougher PEL of the order of $100 \mathrm{meV}$. Moreover, our data show that all clusters exhibit diffusion mechanisms that include both concerted translation and rotation. The overall results of the present study provide key insights for modeling the growth of metal layers and nanostructures on graphene and other van der Waals materials, which is a prerequisite for the directed growth of multifunctional metal contacts in a broad range of enabling devices.
\end{abstract}

\section{Introduction}

Controlled synthesis of thin films and nanostructures from the vapor phase on two-dimensional (2D) materials is essential for leveraging the unique physical attributes of $2 \mathrm{D}$ crystals in a wide array of devices, including photodetectors, catalysts, biosensors, and tunnel field-effect transistors. ${ }^{1-8}$ Vapor-based film growth is a far-from-equilibrium process in which the resulting film morphology is predominantly governed by the kinetic rates of atomic-scale mechanisms. ${ }^{9-11}$ One such mechanism is adatom diffusion on the substrate surface, the rate of which determines the competition among island nucleation and growth and, thereby, largely sets the early-stage film morphology. ${ }^{11}$

In classical homo- and heteroepitaxial growth theory, at temperatures that are relevant for thin-film synthesis experiments, diffusion on the substrate is described as a random walk, during which adatoms perform a thermally activated chain of uncorrelated jumps between neighboring adsorption sites. ${ }^{11}$ We have recently demonstrated, using $a b$ initio and classical molecular dynamics simulations, that the above-described picture is not unconditionally valid during the diffusion of $\mathrm{Ag}, \mathrm{Au}, \mathrm{Pd}, \mathrm{Cu}, \mathrm{Pt}$,

\footnotetext{
${ }^{a}$ Nanoscale Engineering Division, Department of Physics, Chemistry and Biology, Linköping University, SE-58183, Linköping, Sweden. E-mail: zarshenas@gmail.com

${ }^{b}$ Theoretical Physics Division, Department of Physics, Chemistry and Biology, Linköping University, SE-58183, Linköping, Sweden

$\dagger$ Electronic supplementary information (ESI) available. See DOI: 10.1039/ d1cp00522g
}

and $\mathrm{Ru}$ adatoms on single-layer graphene (SLG) at room temperature. ${ }^{12}$ Our results revealed that $\mathrm{Ag}, \mathrm{Au}, \mathrm{Pd}$, and $\mathrm{Cu}$ adatoms experience a markedly flat potential energy landscape (PEL) on the SLG surface, with corrugation of the order of a few tens of meV, which may be significantly modified by vibrational entropy effects. Hence, adatoms follow a super-diffusive pattern known as Lévy walk, in which they move continuously within relatively short-range domains $\left(\sim 1-4 \mathrm{~nm}^{2}\right)$ without being halted by surface adsorption sites, while they occasionally perform long jumps across multiple surface adsorption sites. In contrast, $\mathrm{Pt}$ and $\mathrm{Ru}$ adatoms experience PELs with corrugations of the order of a few hundreds of meV (e.g., comparable with PELs encountered by adatoms during epitaxial growth) and perform random walks.

In the present manuscript, we investigate - employing density functional theory (DFT) calculations and ab initio molecular dynamics (AIMD) simulations - the adsorption energetics, and the room-temperature diffusion dynamics and migration mechanisms of $\mathrm{Ag}, \mathrm{Au}, \mathrm{Cu}$, and Pd dimers, as well as $\mathrm{Ag}$ trimers, on SLG. The study is motivated by the fact that, besides adatoms, multiatomic clusters have been shown to be mobile on a number of substrates, including graphite and graphene. ${ }^{13-20}$ One implication of mobile clusters is that they modify the dynamics governing island nucleation, leading to larger island densities, relative to processes in which adatoms are the only mobile species on the substrate surface. ${ }^{11}$

Our results show that the diffusion pattern followed by the various adspecies is consistent with their interaction strength 
with the SLG substrate and the flatness of the PEL they experience. $\mathrm{Ag}$ (both dimer and trimers), $\mathrm{Au}$ dimers, and $\mathrm{Cu}$ dimers interact relatively weakly with SLG, encounter a PEL which is $\sim 5-40 \mathrm{meV}$ corrugated, and exhibit a super-diffusive behavior. Concurrently, Pd dimers perform a random walk on a considerably rougher PEL with a depth of $\sim 100 \mathrm{meV}$. We also establish that diffusion of $\mathrm{Ag}, \mathrm{Cu}$, and $\mathrm{Au}$ adspecies occurs, primarily, via translation, while they are aligned perpendicularly to SLG, such that only one atom is in contact with the substrate. In contrast, Pd dimers diffuse via in-plane translation and rotation, and they are aligned parallel to SLG with both atoms in the cluster being in constant contact with the substrate. Our overall findings show that cluster diffusion, concerning both dynamics and detailed mechanisms, exhibits non-trivial features that need to be considered when modeling the growth of metals on graphene and other $2 \mathrm{D}$ materials.

\section{Computational methodology}

We perform AIMD simulations to model diffusion of single Ag, $\mathrm{Au}, \mathrm{Cu}$, and Pd dimers, as well as Ag trimers, on SLG within the Vienna $a b$ initio simulation package (VASP). ${ }^{21}$ Core electrons are replaced by the projector augmented wave (PAW) pseudopotentials and the generalized gradient approximation of Perdew, Burke, and Ernzerhof (PBE). ${ }^{22}$ We use an energy cutoff of $400 \mathrm{eV}$ for the plane waves and $\Gamma$-point sampling of the reciprocal space. The SLG substrate is implemented as a hexagonal $6 \times 6$ supercell containing 72 carbon atoms. A vacuum region of $20 \AA$ separates graphene replicas along the $z$-direction. We model the van der Waals forces using the DFT-D3 method with Becke-Johnson (BJ) damping, ${ }^{23}$ which accurately describes the properties of both graphite and diamond. ${ }^{24}$ Simulations are performed accounting for the electronic-spin degrees of freedom for a total time of $1.2 \mathrm{~ns}$ (time step $1 \mathrm{fs}$ ) using canonical sampling (Nosé-Hoover thermostat at $300 \mathrm{~K}$ ) of the configurational space. The simulation output is visualized by the Visual Molecular Dynamics software. ${ }^{25}$

We calculate the lowest energy configuration of the various adspecies on the SGL substrate and we estimate the corrugation of the PEL at $0 \mathrm{~K}$ by computing the ground-state adsorption energies $E_{\text {ads }}^{0 \mathrm{~K}}$ on the hollow, top, and bridge sites using standard DFT structure relaxations and the following expression:

$$
E_{\mathrm{ads}}^{\mathrm{OK}}=E_{0}(\text { Cluster} / \mathrm{SLG})-E_{0}(\text { Cluster })-E_{0}(\mathrm{SLG}) \text {. }
$$

In eqn (1), $E_{0}$ (Cluster/SLG) is the total energy of the cluster/SLG system, $E_{0}$ (Cluster) is the total energy of an isolated cluster and $E_{0}$ (SLG) is the total energy of the single-layer graphene. Due to a higher degree of freedom of clusters, in comparison with adatoms, $E_{\text {ads }}^{\mathrm{OK}}$ are calculated for several different configurations of the clusters (as shown in Table 1). As in AIMD simulations, DFT calculations employ vdW corrections and include electronic-spin relaxation. $\Gamma$-Point sampling of the reciprocal space is used for all calculations. Selected calculations using a finer $3 \times 3 \times 1 k$-point grid showed no appreciable differences in the absolute values of $E_{\mathrm{ads}}^{\mathrm{oK}}$, such that the use of $\Gamma$-point sampling is deemed to provide sufficient accuracy for studying the energetics of the adspecies adsorption on SLG and establishing its correlation with the AIMD simulation data.

\section{Results and discussion}

\subsection{Diffusion dynamics}

We use AIMD simulations to model the migration of single Ag dimers and trimers, Au dimers, Cu dimers, and Pd dimers on the SLG substrate (see Computational methodology section for more details). Fig. 1 displays the diffusion trajectories of the clusters' center of mass (CM) after a simulation time of $1.2 \mathrm{~ns}$. The data corresponding to the Ag dimer (Fig. 1(a)) reveals that this cluster moves over an area of $\sim 91 \mathrm{~nm}^{2}$. Closer inspection of the trajectory shows that the dimer remains confined in domains with sizes in the range $\sim 0.8$ to $\sim 3 \mathrm{~nm}^{2}$ (representative domains are marked with bold lines in Fig. 1(a)) where it diffuses randomly by moving continuously among adsorption sites (see magnified inset in Fig. 1(a), whereby green circles represent the position of carbon atoms in the graphene sheet). Occasionally, it performs directed jumps $\sim 1.5-5.4 \mathrm{~nm}$ long (an example of such jump is marked with a red line in Fig. 1(a)) as it transitions between diffusion domains. The Ag trimer (Fig. 1(b)) exhibits a

Table 1 Static (i.e., $0 \mathrm{~K}$ ) adsorption energies of clusters on SLG. Energies are shifted with reference to the most stable adsorption site for which $E_{\text {ads }}{ }^{0 \mathrm{~K}}=$ 0 . Adsorption energies are calculated for diffusion-relevant cluster alignments with respect to to the substrate as observed in the simulations, i.e., dimer perpendicular to the substrate $(\perp)$, dimer parallel to the substrate $(\|)$, trimer in a configuration where one atom is in contact with the substrates $(\nabla)$, and trimer in a configuration in which two atoms are in contact with the substrate $(\triangle)$. T, B and H denote the top, bridge and hollow sites, respectively. 2NN and $3 \mathrm{NN}$ denote the scenario in which dimer atoms are located on top of carbon atoms which are separated by one and two carbon atoms in a hexagon ring, respectively. The term unstable denotes adsorption sites for which a slight deviation from one configuration leads to a spontaneous change into the other configuration (i.e., $\perp$ to $\|$ or vice versa). In the case of the trimer, the top, bridge and hollow sites correspond to the location of the CM on the SLG surface

\begin{tabular}{|c|c|c|c|c|c|c|c|c|}
\hline Type of cluster & \multicolumn{8}{|c|}{$\underline{E_{\mathrm{ads}}{ }^{\mathrm{oK}}(\mathrm{meV})}$} \\
\hline Ag dimer & 0 & 5 & 60 & 48 & 80 & 67 & 61 & 69 \\
\hline $\mathrm{Cu}$ dimer & 37 & 0 & 233 & Unstable & 660 & Unstable & Unstable & Unstable \\
\hline Pd dimer & Unstable & Unstable & Unstable & 188 & 61 & 153 & 0 & 147 \\
\hline $\mathrm{Ag}$ trimer $(\Delta)$ & 31 & 37 & 0 & & & & & \\
\hline
\end{tabular}



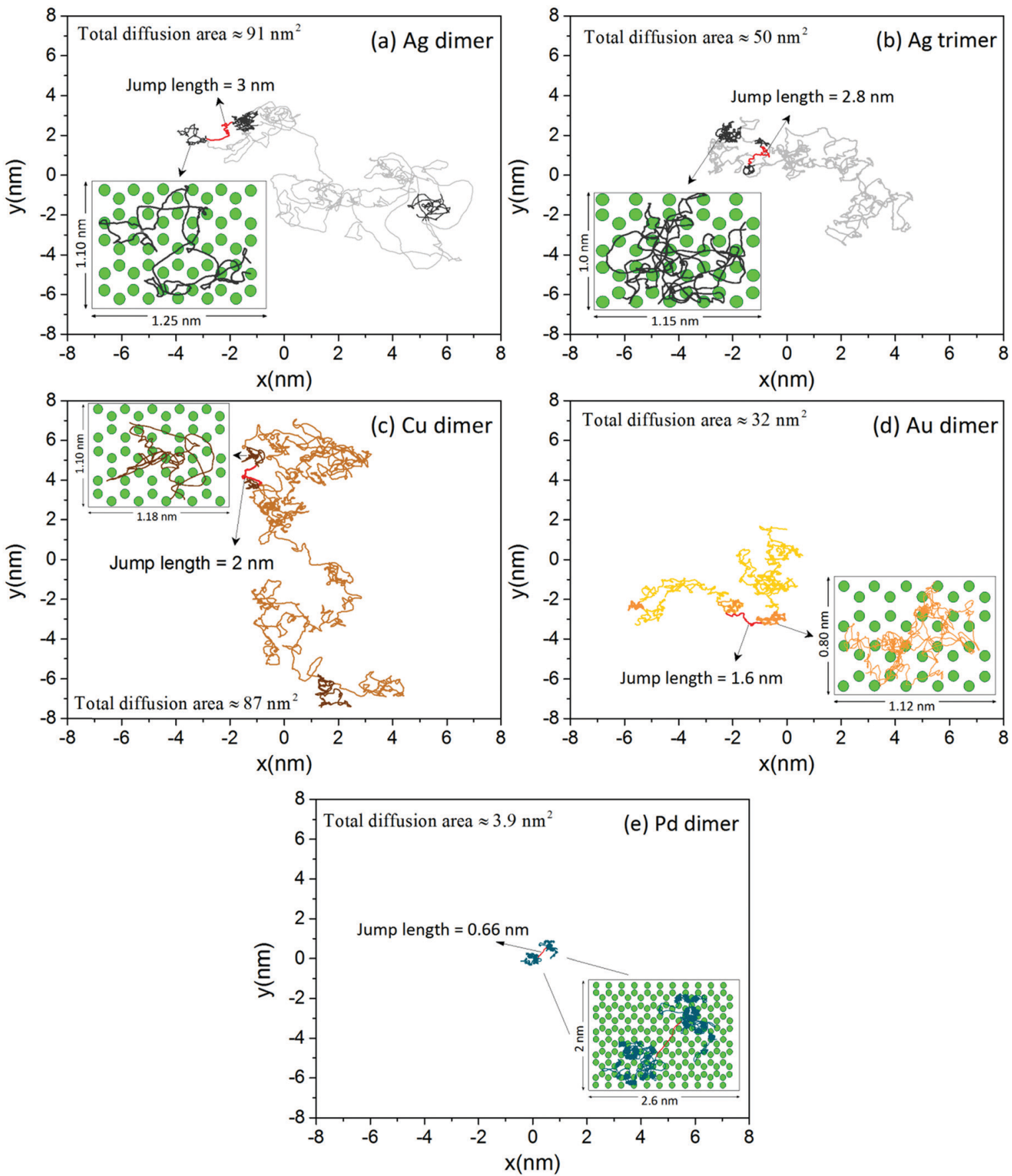

Fig. 1 Diffusion trajectories of (a) Ag dimer, (b) Ag trimer, (c) Cu dimer, (d) Au dimer, and (e) Pd dimer. Representative diffusion domains are marked with bold lines in panels (a) through (d). The jump between two successive domains in each graph in panels (a) through (e) is highlighted with red lines, while the corresponding jump length is also provided. The inset in each panel is a magnified view of a diffusion domain, whereby green circles represent the position of carbon atoms in the graphene sheet. A detailed discussion of the diffusion trajectories and their interpretation is provided in the text.

qualitatively similar trajectory to that of the dimer, with its $\mathrm{CM}$ diffusing in a total area of $\sim 50 \mathrm{~nm}^{2}$ that features $\sim 0.75$ to $2.5 \mathrm{~nm}^{2}$ domains separated by directed jumps $\sim 1.5-3 \mathrm{~nm}$ long. Diffusion patterns consistent with that described for the Ag dimer are also observed for $\mathrm{Cu}$ and $\mathrm{Au}$ dimers (Fig. 1(c) and (d)), respectively; the corresponding total diffusion areas are provided in each figure). Out of three metals, $\mathrm{Au}$ exhibits the shortest diffusion length during 1.2 ns. This is most notably exemplified by the inset in Fig. 1(d), which depicts a typical domain for Au on SLG: diffusion occurs in a smaller area compared to $\mathrm{Ag}$ and $\mathrm{Cu}$ dimers, but still, the motion of the cluster is not halted by a specific adsorption site.

A distinctly different diffusion behavior is obtained for the Pd dimer, as seen in Fig. 1(e). For a total simulation time of $1.2 \mathrm{~ns}$, the CM of this cluster only moves within an area of $\sim 3.9 \mathrm{~nm}^{2}$ and remains confined in well-defined domains 
separated by a jump of $\sim 0.66 \mathrm{~nm}$. Detailed analysis of the cluster trajectory in each of these domains shows that Pd atoms in the dimer mostly lie either on top of the center of adjacent hexagonal rings (i.e., hollow site) or on top of second nearest neighbor carbon atoms, such that the most favorable adsorption site for the CM is the bridge site (see magnified inset in Fig. 1(e)). The analysis also shows that the CM of the Pd dimer resides, on average, for a time of $\sim 75$ ps on each bridge site before transitioning to a neighboring one. This pattern is reminiscent of a random walk, as opposed to the trajectories of the other clusters $(\mathrm{Ag}, \mathrm{Au}$, and $\mathrm{Cu}$ ), which are comprised of correlated jumps across multiple adsorption sites and are, therefore, representative of super-diffusive behavior.

To better understand the diffusion type and dynamics of the various clusters, we calculate from each trajectory displayed in Fig. 1 the time-averaged mean square displacement $\overline{\delta^{2}}(\tau)$ of the cluster CM. This is done for observation time scales equal to $\tau,{ }^{26-29}$ according to the expression: ${ }^{30}$

$$
\overline{\delta^{2}}(\tau)=(1 / N) \sum_{n=0}^{N-1}|x(n \Delta t+\tau)-x(n \Delta t)|^{2}
$$

In eqn (2), $\Delta t$ is the simulation time step, and $N$ represents the total number of sub-trajectories with a duration $\tau$ that can be defined over the total trajectory.

For a simulation time $T$ and an observation time scale $\tau$ consisting of $N_{T}$ and $N_{\tau}$ time steps $\Delta t, N=N_{T}-N_{\tau}+1$. We also note that each $\overline{\delta^{2}}(\tau)$ value corresponds to an average entailing information of all sub-trajectories with a duration $\tau$. From the latter, it follows that the statistical significance of a given $\overline{\delta^{2}}(\tau)$ decreases with increasing $\tau$, since longer $\tau$ values yield a smaller number of sub-trajectories available for calculating $\overline{\delta^{2}}(\tau)$. For that reason, following the methodology proposed by Saxton, ${ }^{30}$ only $\overline{\delta^{2}}(\tau)$ points within an interval $\left[0: \tau_{\text {cut }}\right]$ are considered. Here, we take $\tau_{\text {cut }}=50 \mathrm{ps}$, which corresponds to the mean time during which a cluster remains in a diffusion domain.

Log-log scale $\overline{\delta^{2}}(\tau)$ vs. $\tau$ curves extracted from the simulated trajectories for $\mathrm{Ag}, \mathrm{Au}$, and Cu clusters in Fig. 1(a) through (d) are displayed in Fig. 2. The data show that the $\overline{\delta^{2}}(\tau) v s . \tau$ curves exhibit a log-log slope $\alpha>1$, i.e., $\overline{\delta^{2}}(\tau)$ does not exhibit a linear dependence on time. Using the approach proposed by Kepten et al., ${ }^{31}$ we compute the most probable value of $\alpha$ by fitting linear functions to the $\log$-log curves in Fig. 2 over multiple time intervals (see Fig. S1 in the ESI $\uparrow$ for more details) and find that $\alpha$ ranges between 1.19 and 1.33. The latter quantifies the non-linear correlation among atomic displacement and time, it confirms the super-diffusive nature of the trajectories ${ }^{32-34}$ in Fig. 1(a)-(d), and it is in agreement with the anomalous nature of diffusion for $\mathrm{Ag}, \mathrm{Au}, \mathrm{Cu}$, and Pd adatoms on SLG established in a recent study of ours. ${ }^{12}$ Moreover, based on long ( $\geq 30 \mathrm{~ns}$ ) classical MD simulations of $\mathrm{Ag}$ adatom diffusion on graphene $\mathrm{e}^{12}$ and $\mathrm{Au}_{245}$ cluster diffusion on graphite, ${ }^{35}$ we expect that the $\log$-log slope $\alpha$ will approach unity for $\tau \rightarrow \infty$. This indicates that, at the long-time limit, the motion of adspecies between diffusive domains can be effectively treated as random walk.

For the case of the Pd dimer, the cluster remains trapped for $\sim 75 \mathrm{ps}$ in each adsorption site before diffusing to the next one, i.e., the simulated trajectory of $1.2 \mathrm{~ns}$ contains $\sim 15$ diffusive jumps between neighboring sites and one long jump between diffusion domains. This number of migration events represents a rather small statistical sample for extracting $\overline{\delta^{2}}(\tau) v s . \tau$ curves and computing meaningful values for the exponent $\alpha$, and hence we choose to analyze the trajectory of the Pd dimer only qualitatively.

\subsection{Diffusion mechanisms and adsorption energetics}

Inspection of the simulation movies shows that the dimers may exhibit two stable configurations on the SLG surface: either both atoms in the cluster are in contact with the substrate

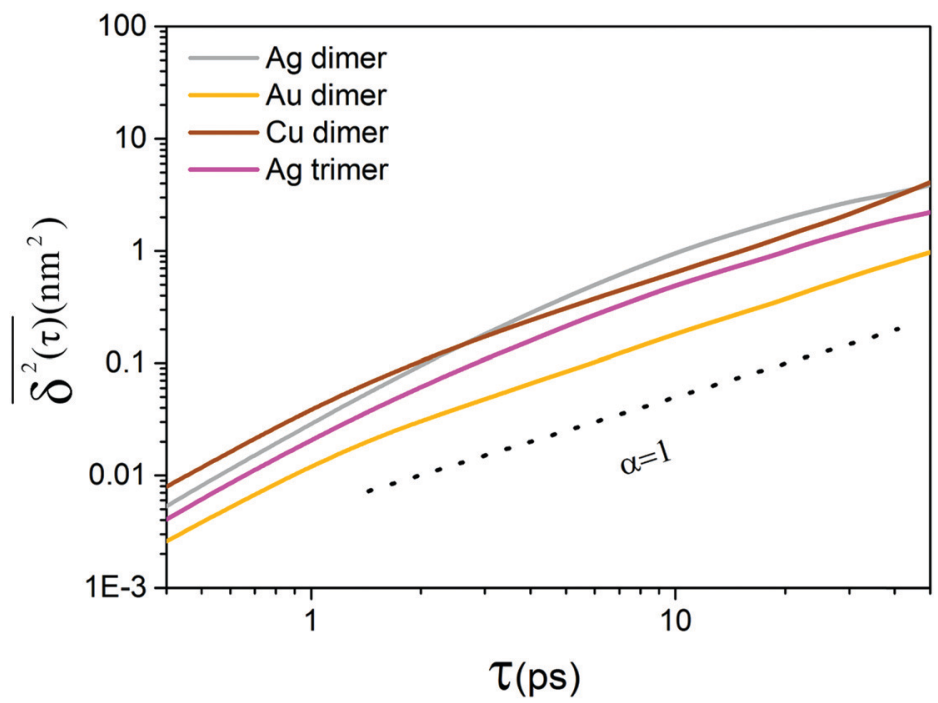

Fig. $2 \overline{\delta^{2}}(\tau)$ vs. $\tau$ curves (plotted in log-log scale) extracted from simulated trajectories for Ag, Au, and Cu adspecies. All curves exhibit slopes $\alpha>1$ (the $\alpha=$ 1 is also plotted as a dashed line for reference), which is consistent with super-diffusive nature of the simulated trajectories, as explained in the text. 
(parallel alignment) or one atom only retains contact with the substrate with the second atom being only in contact with the first one (perpendicular alignment). In a first diffusion mechanism (depicted in Fig. 3(a)) the dimer translates across multiple adsorption sites of the SLG by remaining in the perpendicular configuration. This way of surface migration is observed $100 \%$ of the simulation time for $\mathrm{Cu}$ and Au dimers, and $75 \%$ of the time for Ag. In the remaining $25 \%$ of the simulation time, the $\mathrm{Ag}$ dimer is parallel to the SLG surface and diffuses by translating and occasionally rotating around the axis defined by the substrate surface normal (Fig. 3(b)). The transition between the diffusion modes (perpendicular and parallel) occurs instantly in less than a picosecond, such that this process does not need to be taken into account when calculating the elapsed time for each mechanism. The Pd dimer is aligned parallel to the substrate during the entire simulation time, and diffusion occurs by successive rotations and occasional translations between neighboring bridge sites (see Fig. 3(c)).

Owing to the fact that a trimer exhibits a larger number of degrees of freedom for atomic motion relative to a dimer, more diffusion modes are intuitively expected for the former case. Three stable configurations are, predominantly, observed during the simulation of the $\mathrm{Ag}$ trimer diffusion. The trimer is aligned perpendicularly with either one or two atoms in contact with the substrate, while in the parallel alignment all three atoms retain contact with the SLG surface. With reference to these configurations, four mechanisms are identified during the diffusion of the $\mathrm{Ag}$ trimer on SLG: (i) translation of the trimer with two Ag atoms in contact with the substrate (Fig. 4(a); 87\% of the simulation time); (ii) translation along with rotation around the axis defined by the substrate surface normal (Fig. 4(b); 3\% of the simulation time); (iii) translation of the trimer with one atom in contact with the substrate, along with partial rotation around an axis parallel to the substrate surface (Fig. 4(c); 9\% of the simulation time); and (iv) translation of the trimer with all atoms in contact with the substrate (parallel configuration), along with rotation around defined by the substrate surface normal (Fig. 4(d); 1\% of the total simulation time). Once again, the very short transition time between each mechanism can be ignored when calculating the time of each mechanism.
In our previous simulation study on the diffusion of metal adatoms on graphene, ${ }^{12}$ we demonstrated a correlation among the diffusion type (i.e., normal vs. super-diffusive behavior) and the corrugation of the adatom-SLG PEL. To explore the relevance of this correlation for cluster diffusion investigated in the present work, we calculate (using DFT) the $0 \mathrm{~K}$ values of adsorption energy $E_{\text {ads }}{ }^{\mathrm{OK}}$ of $\mathrm{Ag}, \mathrm{Au}, \mathrm{Cu}$ and $\mathrm{Pd}$ dimers, as well as $\mathrm{Ag}$ trimer in different possible configurations (see eqn (1) in Section 2). The results are listed in Table 1, where all calculated energies are shifted with respect to the corresponding most stable adsorption site for which we set $E_{\mathrm{ads}}{ }^{\mathrm{OK}}=0$. For dimers, energies are computed for the molecular bond axis perpendicularly $(\perp)$ or parallel $(\|)$ to the SLG substrate. Adsorption states denoted as unstable in Table 1 indicate dimer configurations which spontaneously relax to a different configuration upon slight perturbation from the high-symmetry positions. In the case of the Ag trimer, since it spends almost $99 \%$ of the simulation time being perpendicularly aligned to the SLG, only $E_{\text {ads }}{ }^{\text {oK }}$ values that correspond to the most stable perpendicular configurations (among all possible ones) during diffusion are presented in Table 1.

The static (i.e., $0 \mathrm{~K}$ ) adsorption energy calculations for dimers are consistent with the trends observed in the dynamic simulations with respect to the cluster alignment: $\mathrm{Au}$ and $\mathrm{Cu}$ dimers are aligned predominantly perpendicularly to the substrate, Pd remains parallel, while Ag attains both types of alignment. To further understand the correlation among cluster orientation and adsorption energetics, we perform additional AIMD simulations, in which non-favorable cluster alignments are explicitly used as starting configurations, i.e., (i) parallel alignment for $\mathrm{Ag}$ trimers, and $\mathrm{Cu}$ and $\mathrm{Au}$ dimers; and (ii) perpendicular alignment for Pd dimers. Visual inspection of the simulation output shows that all clusters realign to their most common diffusion configuration in less than a few picoseconds after the simulation start. These findings confirm the lowest energy configurations of the clusters established from the $E_{\mathrm{ads}}{ }^{0 \mathrm{~K}}$ calculations listed in Table 1.

The data in Table 1 also show that the $\mathrm{Ag}, \mathrm{Au}$, and $\mathrm{Cu}$ dimers, as well as the Ag-trimer, encounter a PEL with a corrugation (i.e., smallest energy difference between adsorption sites) in the range 5 to $37 \mathrm{meV}$. This relatively flat PEL is

\section{(a)}

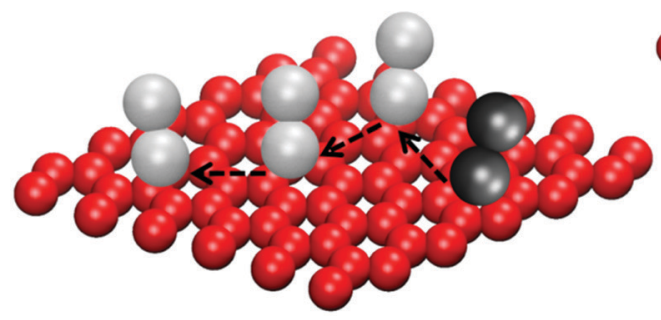

(b)

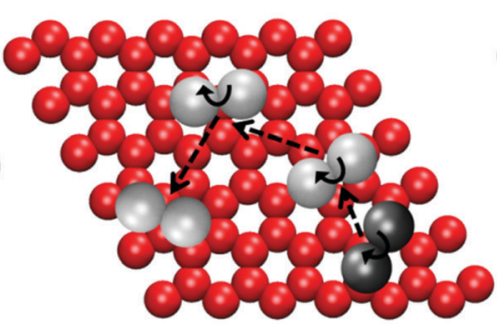

(c)

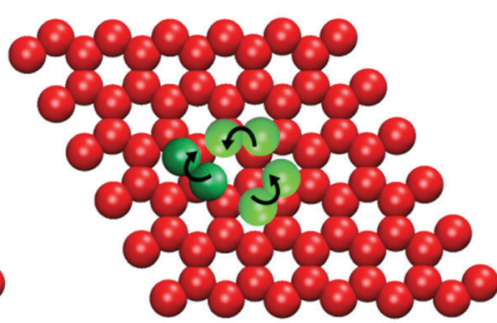

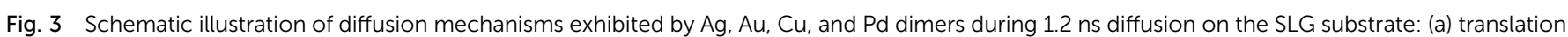

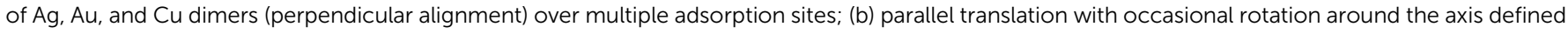

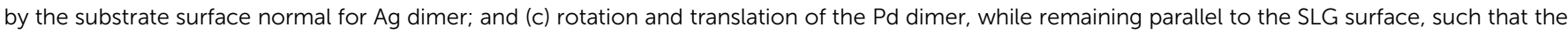

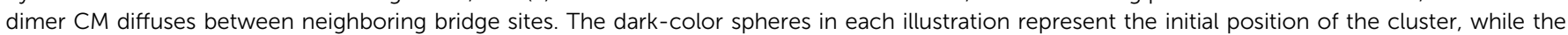
light-color ones show all subsequent positions during diffusion on the SLG substrate. 

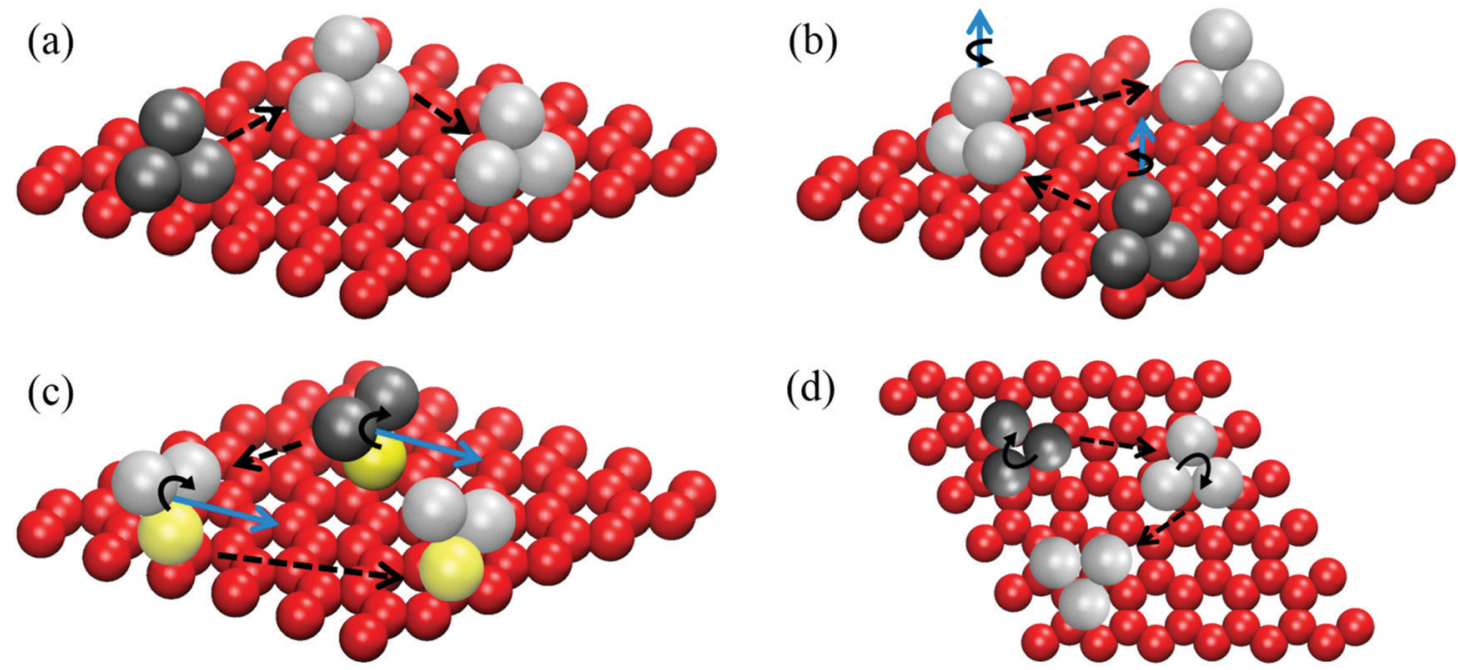

(d)

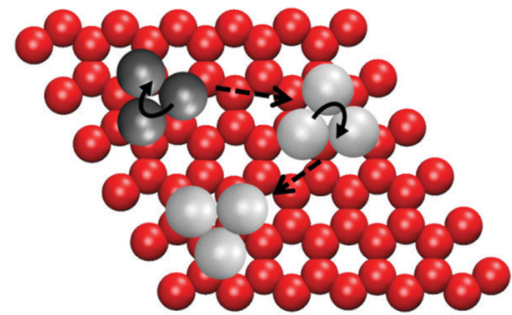

Fig. 4 Diffusion mechanisms exhibited by Ag trimer on SLG during $1.2 \mathrm{~ns}$ of simulation: (a) translation with two Ag atoms in contact with the substrate (perpendicular configuration); (b) translation along with rotation around the axis parallel to the substrate surface normal in perpendicular configuration; (c) translation in perpendicular configuration, with one $\mathrm{Ag}$ atom is in contact with the substrate (yellow-colored sphere) and partial rotation around the axis parallel to the surface; and (d) translation with all the three Ag atoms are in contact with the substrate (parallel configuration) along with rotation around the axis defined by the substrate surface normal. The dark-color spheres in each panel represent the initial position of the cluster, while the light-color ones show all subsequent positions during diffusion on the SLG substrate. The blue arrows indicate the axis around which the cluster rotates.

indicative of weak adspecies-substrate interaction and offers transition pathways that can support the super-diffusive behavior observed in Fig. 1(a) through (d). In contrast, the Pd experiences a significantly rougher PEL (corrugation of the order of $\sim 100 \mathrm{meV}$ ), which is indicative of strong adspeciessubstrate interaction and can explain the random-walk diffusive behavior. The above-described correlation is consistent with our recent findings with regards to the effect of $\mathrm{PEL}$ on the diffusion dynamics of $\mathrm{Ag}, \mathrm{Au}, \mathrm{Pd}, \mathrm{Cu}, \mathrm{Pt}$, and $\mathrm{Ru}$ adatoms on SLG. ${ }^{12}$

\section{Conclusions}

We study via AIMD simulations the diffusion of $\mathrm{Ag}, \mathrm{Au}, \mathrm{Cu}, \mathrm{Pd}$ dimers, and Ag trimers on SLG at room temperature. Visualization of the simulation output and quantitative analysis of the simulated trajectories show that $\mathrm{Ag}, \mathrm{Au}$, and $\mathrm{Cu}$ clusters diffuse by performing correlated jumps over multiple adsorption sites, which is a behavior that can be classified as super-diffusive. In contrast, Pd dimers diffuse between neighboring adsorption sites in a pattern that is reminiscent of random-walk behavior. The differences in the diffusion dynamics can be explained in light of the corrugation of the PEL; $\mathrm{Ag}, \mathrm{Au}$, and $\mathrm{Cu}$ clusters encounter a flat PEL (corrugation of a few tens of meV), while Pd dimers diffuse on a rougher PEL in which the energy difference between adsorption sites is of the order of $\sim 100$ of meV. Moreover, our results indicate complex diffusion mechanisms for all clusters that involve both translation and rotation. $\mathrm{Ag}, \mathrm{Cu}$, and $\mathrm{Au}$ adspecies diffuse by being predominantly perpendicularly oriented to the SLG, such their contact and interaction with the substrate is minimized. In contrast, Pd dimers diffuse via in-plane translation and rotation and both atoms in the cluster remain in contact with the substrate.

Our results show that differences in metal-cluster mobility may be an important aspect for understanding experimental data with regards to the growth of metal layers and nanostructures on graphene and other van der Waals materials. For instance, Zhou et al. have reported that $\mathrm{Au}$ layers exhibit a more pronounced three-dimensional growth morphology on graphene ${ }^{36}$ as compared to Pd which tends to develop flatter and more uniform layers. ${ }^{37}$ Similar trends were also found by Gong et al. ${ }^{5}$ when considering the growth of $\mathrm{Ag}$ and $\mathrm{Au}$ vs. Pd on $\mathrm{MoS}_{2}$. These differences in the obtained morphology point towards a lower island number density during $\mathrm{Ag}$ and $\mathrm{Au}$ growth, as compared to Pd. This may, in turn, be explained by the overall larger diffusivity (augmented by their super-diffusive character) of $\mathrm{Au}$ and $\mathrm{Ag}$ clusters. Furthermore, the present study, in combination with our previous findings on the migration of adatoms on graphene ${ }^{12}$ show that models of diffusion and initial formation stages of film growth on substrates that exhibit flat PELs cannot be unconditionally based on the conventional random walk theory, but rather on more sophisticated approaches which account for the effect of super-diffusive atomic behavior. Moreover, the data presented herein provide further evidence in favor of the potentially universal relevance of super-diffusive atomic motion for driving self-assembly processes at the nanoscale, including formation of three-dimensional $\mathrm{Pb}$ islands on $\mathrm{Si}(111)$ and $\mathrm{Ni}(111)$ substrates,${ }^{38-40}$ and phase separation of $\mathrm{Pb}$ on $\mathrm{Ge}(111){ }^{41}$

\section{Conflicts of interest}

There are no conflicts to declare. 


\section{Acknowledgements}

The authors acknowledge Linköping University ("LiU Career Contract, Dnr-LiU-2015-01510, 2015-2020"), the Swedish research council (contract VR-2015-04630), the ÅForsk foundation (contract ÅF 19-137), and the Olle Engkvist foundation (contract SOEB 190-312) for financial support. Simulations and data handling were enabled by resources provided by the Swedish National Infrastructure for Computing (SNIC) at the National Supercomputer Centre (NSC) partially funded by the Swedish Research Council through Grant Agreement No. VR-2015-04630.

\section{References}

1 T. J. Echtermeyer, S. Milana, U. Sassi, A. Eiden, M. Wu, E. Lidorikis and A. C. Ferrari, Surface Plasmon Polariton Graphene Photodetectors, Nano Lett., 2016, 16, 8-20.

2 T. Mueller, F. Xia and P. Avouris, Graphene photodetectors for high-speed optical communications, Nat. Photonics, 2010, 4, 297-301.

3 Y. Xu, C. Hsieh, L. Wu and L. K. Ang, Two-dimensional transition metal dichalcogenides mediated long range surface plasmon resonance biosensors, J. Phys. D: Appl. Phys., 2019, 52, 065101.

4 X. Liu, Y. Han, J. W. Evans, A. K. Engstfeld, R. J. Behm, M. C. Tringides, M. Hupalo, H. Lin, L. Huang, K. Ho, D. Appy, P. A. Thiel and C. Wang, Growth morphology and properties of metals on graphene, Prog. Surf. Sci., 2015, 90, 397-443.

5 C. Gong, C. Huang, J. Miller, L. Cheng, Y. Hao, D. Cobden, J. Kim, R. S. Ruoff, R. M. Wallace, K. Cho, X. Xu and Y. J. Chabal, Metal Contacts on Physical Vapor Deposited Monolayer $\mathrm{MoS}_{2}$, ACS Nano, 2013, 7, 11350-11357.

6 D. Deng, K. S. Novoselov, Q. Fu, N. Zheng, Z. Tian and X. Bao, Catalysis with two-dimensional materials and their heterostructures, Nat. Nanotechnol., 2016, 11, 218-230.

7 Y. Zhu, L. Peng, Z. Fang, C. Yan, X. Zhang and G. Yu, Structural Engineering of 2D Nanomaterials for Energy Storage and Catalysis, Adv. Mater., 2018, 30, 1-19.

8 T. A. Shifa, F. Wang, Y. Liu and J. He, Heterostructures Based on 2D Materials: A Versatile Platform for Efficient Catalysis, Adv. Mater., 2019, 31, 1804828.

9 P. M. Martin, Handbook of Deposition Technologies for Films and Coatings, William Andrew, 2010.

10 I. Petrov, P. B. Barna, L. Hultman and J. E. Greene, Microstructural evolution during film growth, J. Vac. Sci. Technol., A, 2017, 117, S117.

11 J. Michely and T. Krug, Islands, Mounds and Atoms, Springer, Berlin, 2004.

12 V. Gervilla, M. Zarshenas, D. G. Sangiovanni and K. Sarakinos, Anomalous versus Normal RoomTemperature Diffusion of Metal Adatoms on Graphene, J. Phys. Chem. Lett., 2020, 11, 8930-8936.

13 A. Jamnig, D. G. Sangiovanni, G. Abadias and K. Sarakinos, Atomic-scale diffusion rates during growth of thin metal films on weakly-interacting substrates, Sci. Rep., 2019, 9, 6640 .

14 S. C. Wang, U. Kürpick and G. Ehrlich, Surface Diffusion of Compact and Other Clusters: Ir x on $\operatorname{Ir}(111)$, Phys. Rev. Lett., 1998, 81, 4923-4926.

15 L. Bardotti, A. Hoareau, M. Treilleux, B. Cabaud, A. Perez and F. C. Santos, Diffusion and aggregation of large antimony and gold clusters deposited on graphite, Surf. Sci., 1996, 367, 276-292.

16 U. Kürpick, B. Fricke and G. Ehrlich, Diffusion mechanisms of compact surface clusters: Ir7 on $\operatorname{Ir}(111)$, Surf. Sci., 2000, 470, L45-L51.

17 S. Y. Krylov, Surface Gliding of Large Low-Dimensional Clusters, Phys. Rev. Lett., 1999, 83, 4602-4605.

18 I. M. Goldby, L. Kuipers, B. von Issendorff and R. E. Palmer, Diffusion and aggregation of size-selected silver clusters on a graphite surface, Appl. Phys. Lett., 1996, 69, 2819.

19 L. J. Lewis, Diffusion of gold nanoclusters on graphite, Phys. Rev. B: Condens. Matter Mater. Phys., 2000, 61, 84-90.

20 W. D. Luedtke and U. Landman, Slip Diffusion and Lévy Flights of an Adsorbed Gold Nanocluster, Phys. Rev. Lett., 1999, 82, 3835-3838.

21 G. Kresse and J. Hafner, Ab. initio molecular dynamics for liquid metals, Phys. Rev. B: Condens. Matter Mater. Phys., 1993, 47, 558.

22 J. P. Perdew, K. Burke and M. Ernzerhof, Generalized gradient approximation made simple, Phys. Rev. Lett., 1996, 77, 3865-3868.

23 S. Grimme, S. Ehrlich and L. Goerigk, Effect of the Damping Function in Dispersion Corrected Density Functional Theory, J. Comput. Chem., 2011, 32, 1456-1465.

24 I. Mosyagin, D. Gambino, D. G. Sangiovanni, I. A. Abrikosov and N. M. Caffrey, Effect of dispersion corrections on ab initio predictions of graphite and diamond properties under pressure, Phys. Rev. B, 2018, 98, 174103.

25 W. Humphrey, A. Dalke and K. Schulten, VMD: Visual Molecular Dynamics, J. Mol. Graphics, 1996, 14, 33-38.

26 H. Qian, M. P. Sheetz and E. L. Elson, Single particle tracking Analysis of diffusion and flow in two-dimensional systems, Biophys. J., 1991, 60, 910-921.

27 M. J. Saxton, Anomalous Diffusion Due to Obstacles: A Monte Carlo Study, Biophys. J., 1994, 66, 394-401.

28 M. J. Saxton, Anomalous Diffusion Due to Binding: A Monte Carlo Study, Biophys. J., 1996, 70, 1250-1262.

29 D. V. N. Jr, J. F. Hancock and K. Burrage, Sources of Anomalous Diffusion on Cell Membranes: A Monte Carlo Study, Biophys. J., 2007, 92, 1975-1987.

30 M. J. Saxton, Single-Particle Tracking: The Distribution of Diffusion Coefficients, Biophys. J., 1997, 72, 1744-1753.

31 E. Kepten, A. Weron, G. Sikora, K. Burnecki and Y. Garini, Guidelines for the Fitting of Anomalous Diffusion Mean Square Displacement Graphs from Single Particle Tracking Experiments, PLoS One, 2015, 10, 1-10.

32 R. Metzler and J. Klafter, The random walk's guide to anomalous diffusion: a fractional dynamics approach, Phys. Rep., 2000, 339, 1-77. 
33 F. Höfling and T. Franosch, Anomalous transport in the crowded world of biological cells, Rep. Prog. Phys., 2013, 76, 046602.

34 E. Barkai, Y. Garini and R. Metzler, Strange kinetics of single molecules in living cells, Phys. Today, 2012, 65, 29.

35 Y. Maruyama and J. Murakami, Truncated Lévy walk of a nanocluster bound weakly to an atomically flat surface: Crossover from superdiffusion to normal diffusion, Phys. Rev. B: Condens. Matter Mater. Phys., 2003, 67, 1-5.

36 H. Zhou, C. Qiu, Z. Liu, H. Yang, L. Hu, J. Liu, H. Yang, C. Gu and L. Sun, Thickness-dependent morphologies of gold on Nlayer graphenes, J. Am. Chem. Soc., 2010, 132, 944-946.

37 H. Zhou, F. Yu, H. Yang, C. Qiu, M. Chen, L. Hu, Y. Guo, H. Yang, C. Gu and L. Sun, Layer-dependent morphologies and charge transfer of $\mathrm{Pd}$ on n-layer graphenes, Chem. Commun., 2011, 47, 9408-9410.

38 M. Hupalo and M. C. Tringides, Ultrafast kinetics in $\mathrm{Pb} /$ $\mathrm{Si}$ (111) from the collective spreading of the wetting layer, Phys. Rev. B: Condens. Matter Mater. Phys., 2007, 75, 1-7.

39 T. R. J. Bollmann, R. Van Gastel, H. J. W. Zandvliet and B. Poelsema, Anomalous decay of electronically stabilized lead mesas on Ni(111), Phys. Rev. Lett., 2011, 107, 1-5.

40 K. L. Man, M. C. Tringides, M. M. T. Loy and M. S. Altman, Superdiffusive motion of the $\mathrm{Pb}$ wetting layer on the $\mathrm{Si}(111)$ surface, Phys. Rev. Lett., 2013, 110, 1-5.

41 Y. Sato, S. Chiang and N. C. Bartelt, Spontaneous domain switching during phase separation of $\mathrm{Pb}$ on $\mathrm{Ge}(111)$, Phys. Rev. Lett., 2007, 99, 1-4. 